\title{
The effect of strain history on dynamic necking in titanium alloys, experimental and numerical analysis
}

\author{
Govind Gour ${ }^{1 *}$, Daniel Thomson ${ }^{1}$, Julian Reed ${ }^{1}$, Nik Petrinic $^{1}$, and Antonio Pellegrino ${ }^{1}$ \\ ${ }^{1}$ Department of Engineering Science, University of Oxford, Parks Road, OX1 3PJ, United Kingdom
}

\begin{abstract}
The determination of the dynamic response of titanium alloys is vital for aerospace industrial applications. The present research investigates the effect of strain history and temperature on the dynamic strain localization in Ti-6Al-4V grade-5 alloy. The experimental campaign was conducted using a Split Hopkinson Tensile Bar (SHTB) equipped with an ultra-highspeed camera. The characterization of the material is highly affected once necking occurs. The true dynamic response is compared for various loading conditions. Furthermore, in order to provide insights about on the local dynamic behavior, a numerical model is established using LSDYNAExplicit solver for monotonic loading conditions. Different constitutive material models are compared, including the phenomenological material model Johnson-Cook, and physically based model, Modified BammanChiesa-Johnson (BCJ).
\end{abstract}

\section{Introduction}

Titanium alloys, with their high strength to weight ratio coupled with high corrosion resistance, have been popular for the design of aviation engine components such as fan blades and casings. In particular, Ti-alloys contribute $25 \%$ of the overall weight of modern jet engines in the form of outer casing, fan blades, and low-pressure compressor [1]. Dynamic events such as bird strike and blade release require the understanding of the behaviour of lightweight engineering materials at high rates of deformation. The mechanical response of Ti-alloys is usually measured using the Split Hopkinson Bar apparatus (SHTB). The displacements and forces on the specimen can be determined from the recorded strain gauge signals using one dimensional wave analysis and used to calculate nominal stresses and strains within the specimen. Local deformations are observed using ultra-high-speed cameras.

However, tensile dynamic testing poses limitations such as the poor estimation of the equivalent stress based on the logarithmic form of true curves [2]. In ductile engineering alloys, once necking is initiated, the average estimation of the reduced cross-section within the specimen gauge length induces errors $[3,4]$. In the dynamic loading condition, the necking becomes substantially localised and approximation of the true stress and true strain

*Corresponding author: govind.gour@eng.ox.ac.uk 
in the necked region. It is only possible by measuring precise measurements of the diameter evolution.

The present investigation explores the effect of strain history and the necking evolution of titanium alloys. The evolution of nominal and effective strain rates was measured and compared using digital image correlation. Finally, a numerical analysis has been performed using the LSDYNA-Explicit ${ }^{\circledR}$ solver for monotonic loading conditions, where different materials models were assessed and compared.

\section{Experimental apparatus and results}

The following section describes the experimental setup used to investigate the high strainrate loading of pristine and pre-strained Ti-64 cylindrical samples.

\subsection{Experimental apparatus}

The experimental investigation was performed using cylindrical specimens with a gauge length of $3 \mathrm{~mm}$ in diameter and $8 \mathrm{~mm}$ in length, as shown in Fig. 1. Three batches of the Ti64 alloy specimens were strained at quasi-static loading rates to $6 \%, 10 \%$ and $14 \%$ using screw driven Zwick 050 mechanical tensile machine. The strain was controlled using a video extensometer.

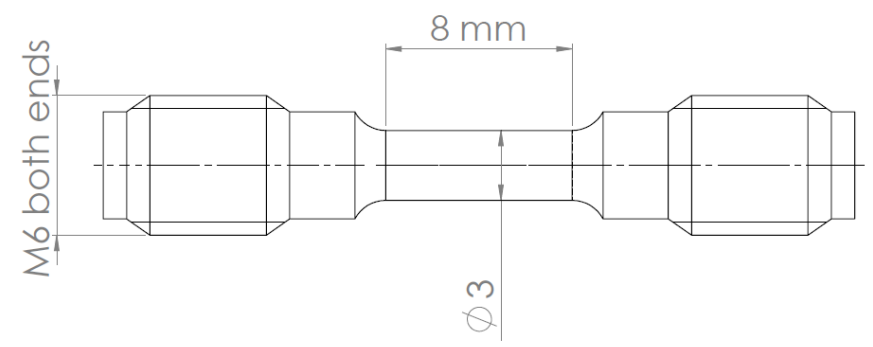

Fig. 1. Cylindrical specimen for the quasi-static and high-rate monotonic loading investigation.

Pre-strained samples were later reloaded in Split-Hopkinson Bar apparatuses (SHB) for high-rate characterization (e.g. $10^{2}-10^{3} \mathrm{~s}^{-1}$ ). In the SHB system, the specimen is placed in between input and output bars and then a stress pulse is generated using a striker. The pulse travels through the input bar, the specimen, and the output bar. The recorded response of the specimen depends on the magnitude, duration, and shape of the generated stress pulse. The experiment is also supported by high-speed imaging to measure the strain localization under the dynamic loading. Fig. 2 presents the basic layout the experimental setup of the SHB which is able to generate tensile deformation ranging from $10^{2} \mathrm{~s}^{-1}$ to $10^{4} \mathrm{~s}^{-1}$ with a pulse duration equal to $1 \mathrm{~ms}$.

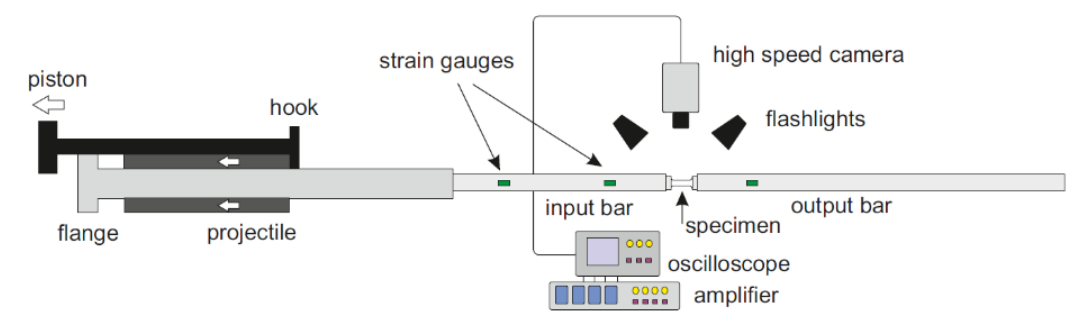

Fig. 2. Basic layout of the Split Hopkinson Tensile Pressure Bar at the University of Oxford Impact Engineering Lab. 
The nominal response (engineering stress-strain) is estimated using recorded strain gauge. However, high-rate tensile experiments on ductile alloys introduce additional technical difficulties. Because of necking, deformations cease to be uniform along the gauge length. Consequently, the computation of stresses and strains using their nominal or logarithmic form poorly approximates the true stress and true strain in the necked region of the specimen $[3,4]$. Moreover, post necking strain is localized and cannot be related to gauge length for stress computation. A high-speed camera is employed to capture the sequence of the specimen's deformation and later digital image correlation (DIC) technique is employed to evaluate the diameter reduction. The accurate measurement of the diameter reduction aids in estimating the true stress-strain under the rapid deformation.

Fig. 3 illustrates the comparison of the diameter evolution for the effect of the quasistatic pre-strain on the high rate response of the Ti-64 alloy. The final diameter for the prestrained cases are found to be higher than pristine monotonic loading condition. It is noticed that pre-straining increases the failure engineering strain ranging from $20 \%$ to $25 \%$. Under high rate deformation, the material experience rise in the temperature (adiabatic heating) is expected to influence the necking evolution. While for the pre-strain case, this temperature is expected to lower than the monotonic cases.

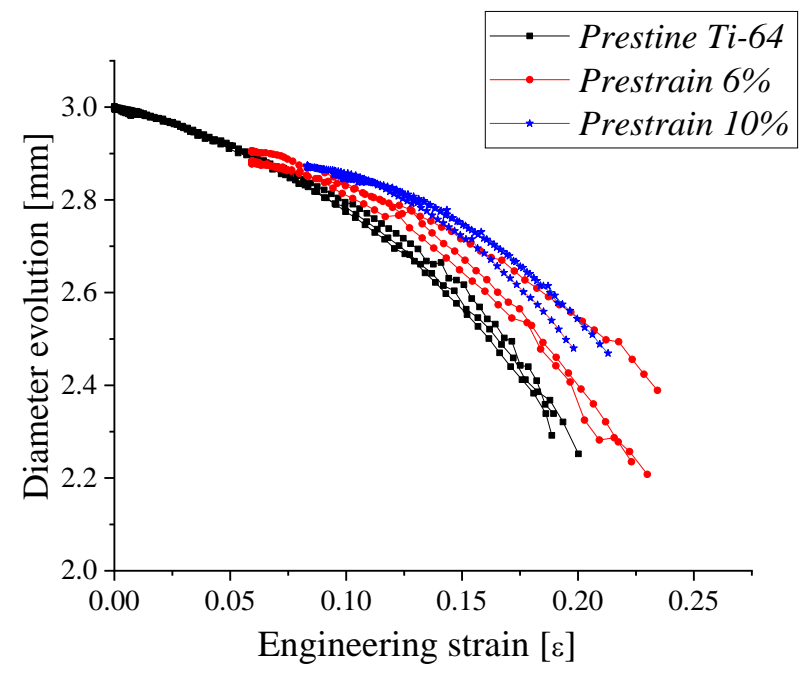

Fig. 3. Diameter reduction of the pristine and pre-strain Ti-64 cylindrical specimens.

\section{Numerical modelling and results}

Following the obtained experimental results, further investigation of the dynamic strain localization is conducted using commercial LSDYNA-Explicit solver, and available in-house and built-in LSDYNA material models are compared.

\subsection{Development of numerical model}

A three-dimensional numerical Finite Element (FE) model was developed using under integrated 8-node solid elements, see Fig. 4. The model is simplified and only specimen geometry is considered for numerical analysis. The experimental loading conditions are applied by keeping one of the ends fixed while the extracted velocity profile from the DIC analysis of the experiments is applied on the other end of the specimen. Material modelling requires to capture the combined effect of strain hardening, strain rate and temperature 
influence on the Ti-64. In the present analysis, a Modified Johnson-Cook, a Tabulated Johnson-Cook and a physically-based BCJ model have been used to compare their predictive capabilities. These types of models are popular due to their relatively easy implementation in the finite element framework and reduction in the computational time. The latter aspect is a crucial factor for high deformation events, as this requires a small time step to capture the material response.

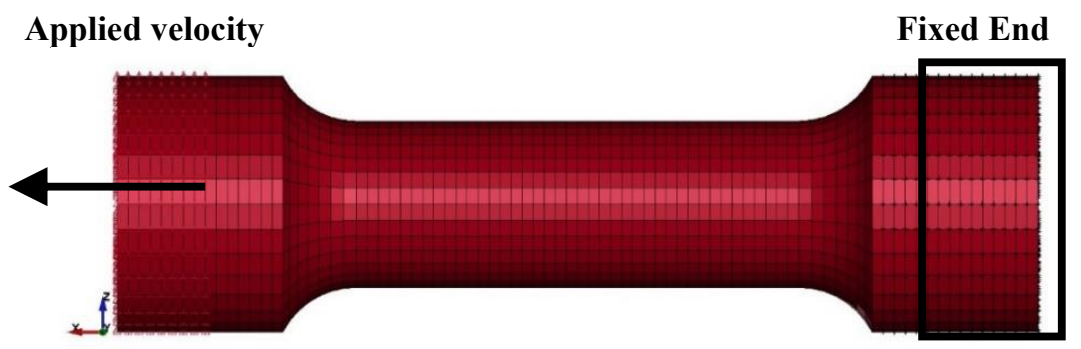

Fig. 4. Three-dimensional cylindrical numerical model with LSDYNA-Explicit ${ }^{\circledR}$

The Modified JC (MAT-107) parameters are estimated from quasi-static and high-rate experimental datasets of the Ti-64 alloy and key parameters are tabulated in the in Table 1 . This formulation includes adiabatic heating to account for the heat generation during highrate deformation, and Voce-hardening parameters to predict the hardening behaviour. Additionally, the numerical analysis also included a Tabulated Johnson-Cook model (MAT224-GYS) which is based on $J 3$ and able to account for the asymmetric response in the titanium alloys. The parameters tables of MAT-224-GYS are adopted from S. Haight et al. [5]. Finally, Bammann-Chiesa developed a constitutive material model based on the dislocation mechanism [6,7] in the framework of the internal state variables (ISV). The basic formulation was developed for isotropic, hypo-elastic, visco-plastic and void growth damage. In recent years, the model has been improved and multi-mode damage parameters are equipped to predict accurate failure modes. The parameters for modified BCJ model is taken from [8].

Table 1: Modified Johnson-Cook parameters

\begin{tabular}{|c|c|c|c|}
\hline \multicolumn{4}{|c|}{ Modified Johnson-Cook } \\
\hline Density $\rho\left(\right.$ ton $\left./ \mathbf{m m}^{3}\right)$ & Poisson's ratio & \multicolumn{2}{|c|}{ Young's modulus (GPa) } \\
\hline $4.43 \mathrm{e}^{-9}$ & 0.30 & \multicolumn{2}{|c|}{110} \\
\hline Yield strength A (GPa) & Strain rate sensitivity $(C)$ & \multicolumn{2}{|c|}{ Thermal softening (m) } \\
\hline 0.981 & $2.076 \mathrm{e}^{-2}$ & \multicolumn{2}{|c|}{0.68} \\
\hline \multicolumn{4}{|c|}{ Voce-hardening parameters } \\
\hline $\mathbf{Q}_{1}$ & 307.75 & $\mathbf{Q}_{2}$ & 148.789 \\
\hline $\mathbf{C}_{1}$ & 5.502 & $\mathbf{C}_{2}$ & 2.584 \\
\hline $\begin{array}{c}\text { Taylor-Quinney coefficient } \\
(\chi)\end{array}$ & \multicolumn{3}{|c|}{0.95} \\
\hline
\end{tabular}




\subsection{Numerical results and discussion}

The validity of the mesh and application of the boundary conditions have been assessed against the high-rate tensile experimental dataset of the Ti-64 alloys performed using SHB. Fig. 5 presents the comparison of the aforementioned material models and it indicates that Modified BCJ is closely following the global response. On the other hand, phenomenological models are also agree well with monotonic response of the Ti-64.

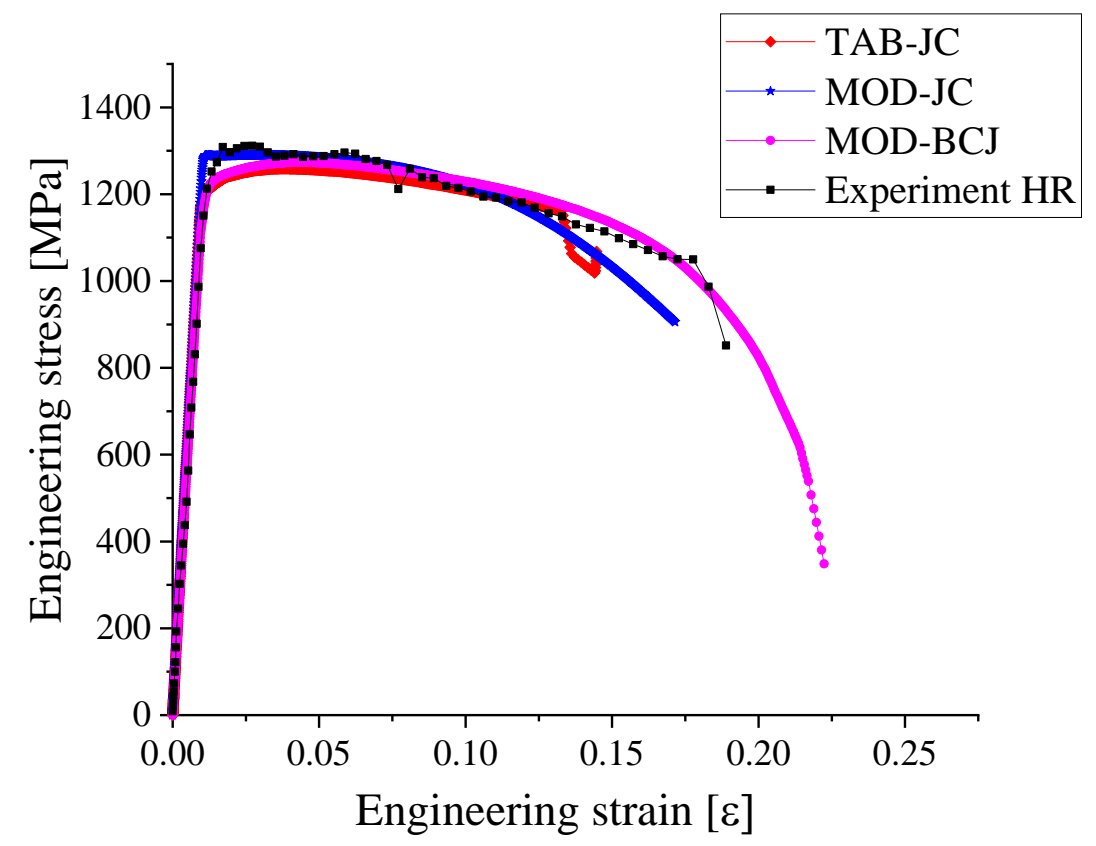

Fig. 5. Comparison of the various numerical models against the high-rate engineering response of Ti64.

The prediction capability of the material models for localized strain are compared to the local behaviour. The final diameter (just before failure) is compared with experiments in Fig. 6. This further affirms that the physically based BCJ formulation is able to follow localized behaviour closely. With the calibrated parameters, the modified JC model (with capability of the adiabatic heating and Voce-hardening coefficient) is also able to predict final diameter within an acceptable error range. Whereas, the tabulated JC model is found to be unable to follow the localized dynamic straining. For comparison of the dynamic straining, it should be noted that duration of the simulations and total displacements applied were adopted from the experimental measurements.

Finally, a multi-step analysis procedure was adopted to introduce the effects of the quasistatic pre-strain into the model for the following dynamic analysis by initialising the stress, strain and state variables from the initial static step within the high-rate model. Fig. 7 presents the comparison of the engineering response of pre-strain and high-rate loading of Ti64 experimental and numerical models. It should be noted that only $10 \%$ pre-strain comparison is presented in the current study. 


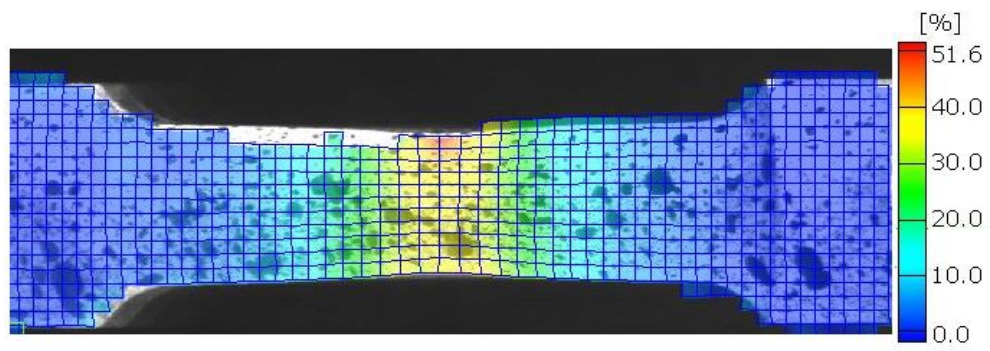

Experimental HR $-2.32 \mathrm{~mm}$

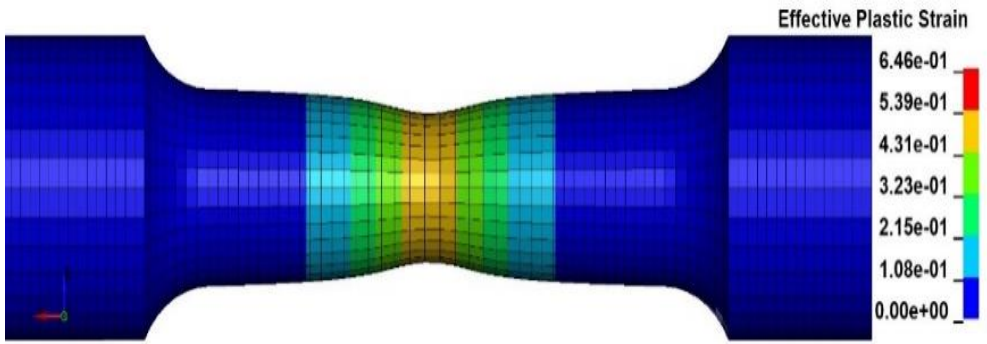

Modified Johnson-Cook $-2.25 \mathrm{~mm}$

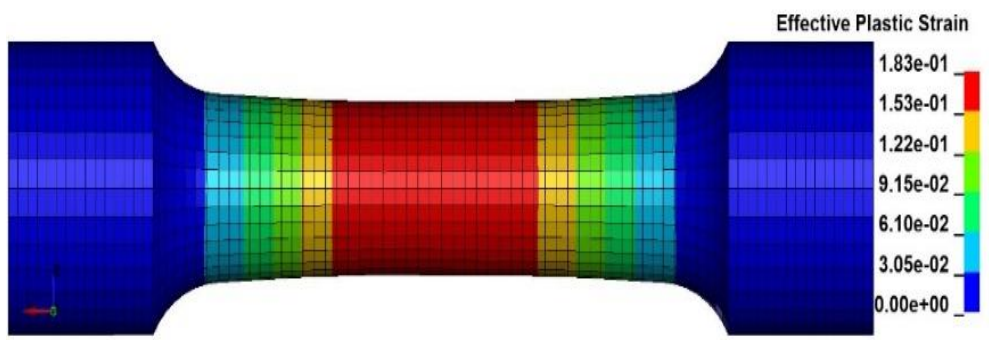

Tabulated Johnson-Cook $-2.74 \mathrm{~mm}$

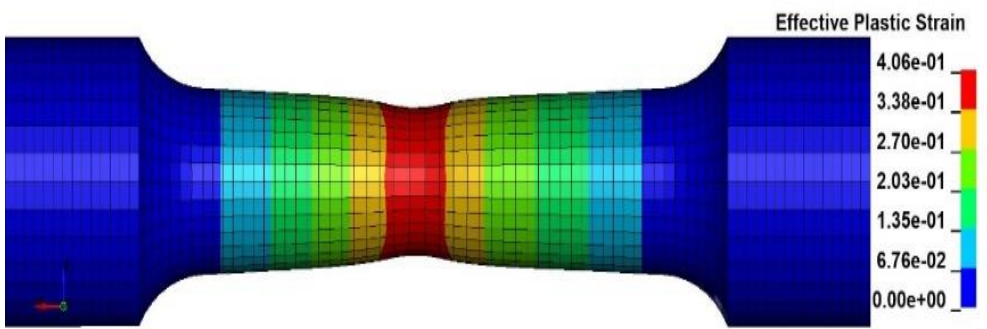

Modified BCJ $-2.35 \mathrm{~mm}$

Fig. 6. Strain localization prediction using Modified Johnson-Cook, Tabulated Johnson-Cook, and Modified BCJ for the high-rate monotonic loading conditions.

The numerical analysis with various material models indicate that, with adequate material parameters, they are all able to describe high-rate engineering response of pristine and $10 \%$ pre-strained Ti-64 tensile specimens with reasonable accuracy. However, the tabulated JC model, which is extensively calibrated for different strain rates and temperatures, predicts inaccurate strain hardening in the pre-strained material (experiments indicated declined hardening behaviour). In addition, even though this model was able to describe the high-rate response in terms of engineering stress and strain with relative accuracy, its inability to capture the localisation and necking effect indicates that in terms of true stress and true strain the results may be significantly worsened. 


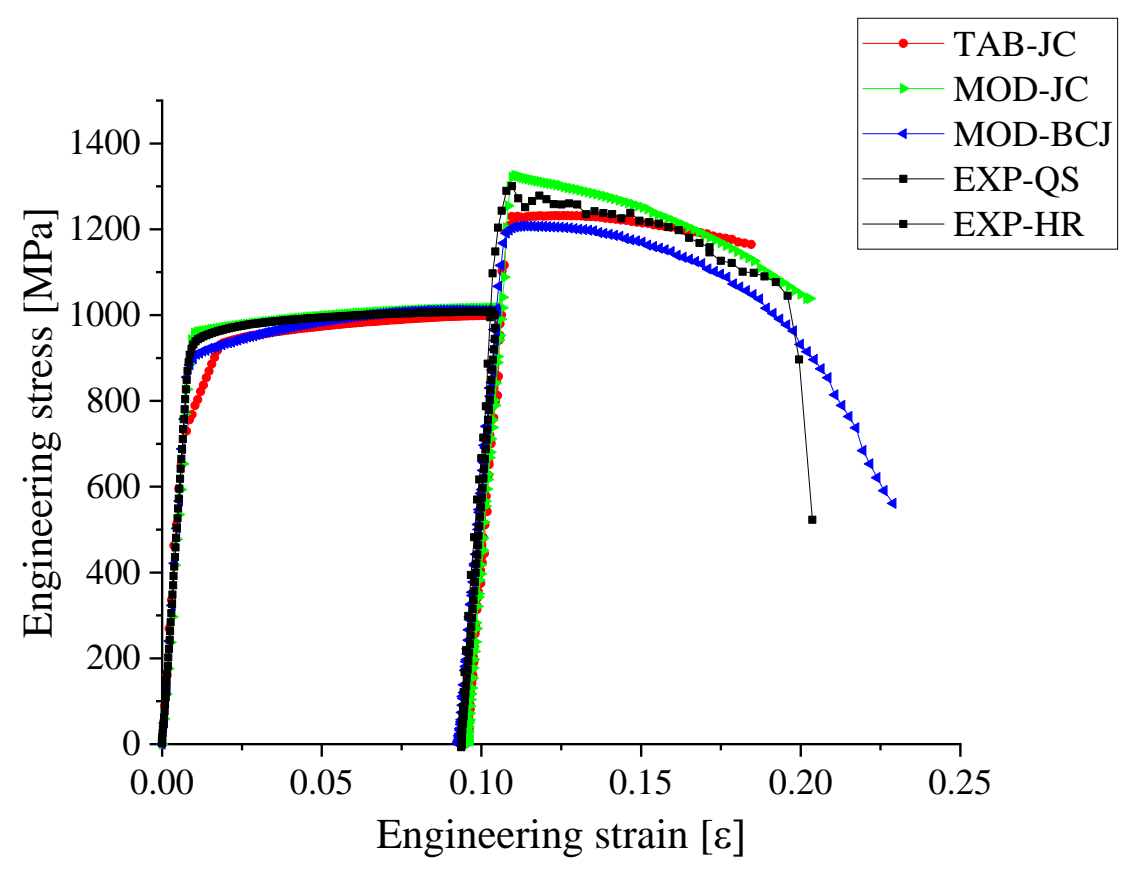

Fig. 7. Comparison of the experimental and numerical results using phenomenological Models for $10 \%$ pre-strained.

On the other hand, the modified JC and the BCJ models both gave much more accurate predictions of the hardening behaviour, even after $10 \%$ pre-strain, and of the diameter reduction in the necking region. The modified JC model explains this behaviour though accounting for adiabatic heating and voce hardening behaviour. On the other hand, the modified BCJ model includes competing damage mechanisms (predominantly void growth damage in the case of tension) in addition to isotropic viscoplastic behaviour, and appears to give a similarly accurate prediction of the HR material response after $10 \%$ pre-strain (Modified JC slightly over predicts the yield stress while BCJ slightly under predicts it). The accuracy of these two models then raises the question of whether the strain history, strainrate, and temperature effects on the material response of Ti-64 can be attributed entirely to viscoplastic hardening plasticity with adiabatic heating effects, or if the void growth damage mechanism included in the BCJ model may also play a key role. Furthermore, the numerical analysis will be used to examine the stress state of the necked zone to evaluate the effect of the pre-straining on the true stress and strain, and additional experimental work is underway to evaluate these models under different temperatures and amounts of pre-strain, including investigation into the potential void growth damage mechanism under these different conditions.

\section{Conclusions}

- The present study investigates the influence of the quasi-static pre-straining on the high-rate performance of the Ti-64. Various material models are used to compare their prediction capability.

- Modified Johnson-Cook and in-house modified BCJ models are found to be closely predicting the engineering response and localised necking of the specimen. 
- Further experiments with different prestrain level and related numerical work is ongoing for conclusive investigation.

\section{Acknowledgement}

The research is sponsored by EPSRC and Rolls-Royce plc as part of the Prosperity Partnership (Cornerstone) project. The authors would like to thank Mr Stuart Carter and $\mathrm{Mr}$ Jeffrey Fullerton for manufacturing the specimens and Dr David Townsend for helpful suggestions for conducting prestrain experiments.

\section{References}

1. Williams, G.L.J.C., Titanium. 2003, Berlin: Springer.

2. Choung, J.M. and S.R. Cho, Study on true stress correction from tensile tests. Journal of Mechanical Science and Technology, 2008. 22(6): p. 1039-1051.

3. Mirone, G., The dynamic effect of necking in Hopkinson bar tension tests. Mechanics of Materials, 2013. 58: p. 84-96.

4. Mirone, G., D. Corallo, and R. Barbagallo, Experimental issues in tensile Hopkinson bar testing and a model of dynamic hardening. International Journal of Impact Engineering, 2017. 103: p. 180-194.

5. Haight, S., et al., Development of a titanium alloy Ti-6Al-4V material model used in $L S$-DYNA. George Mason University, The George Washington University, NASA Glenn Research Center, Washington, DC, Report, 2016.

6. Bammann, D.J. and E.C. Aifantis, A model for finite-deformation plasticity. Acta Mechanica, 1987. 69(1): p. 97-117.

7. Bammann, D.J. and E.C. Aifantis, A damage model for ductile metals. Nuclear Engineering and Design, 1989. 116(3): p. 355-362.

8. Cousins, B.T.s., Development of improved numerical techniques for high strain rate deformation behaviour of titanium alloys, in Constitutive modelling of Ti-6Al-4V alloys for anisotropic, pressure dependent, damage mechanics at high strain rates. 2016, University of Oxford. 